\title{
Bovine Serum Albumin Adsorption in Mesoporous Titanium Dioxide: Pore Size and Pore Chemistry Effect
}

Chang Liu $^{* 1,2,3}$, Yanhua Guo ${ }^{1}$, Qiliang Hong ${ }^{1}$, Chao Rao ${ }^{1}$, Haijuan Zhang ${ }^{1}$, Yihui Dong ${ }^{1}$, Liangliang Huang ${ }^{2 *}$, Xiaohua $\mathrm{Lu}^{1}$, Ningzhong $\mathrm{Bao}^{3}$

1 College of Chemical Engineering, Nanjing Tech University, Nanjing 210009, China 2 School of Chemical, Biological \& Materials Engineering, University of Oklahoma, Norman, 73019, United States

3 Jiangnan Graphene Research Institute, Changzhou 213149, China

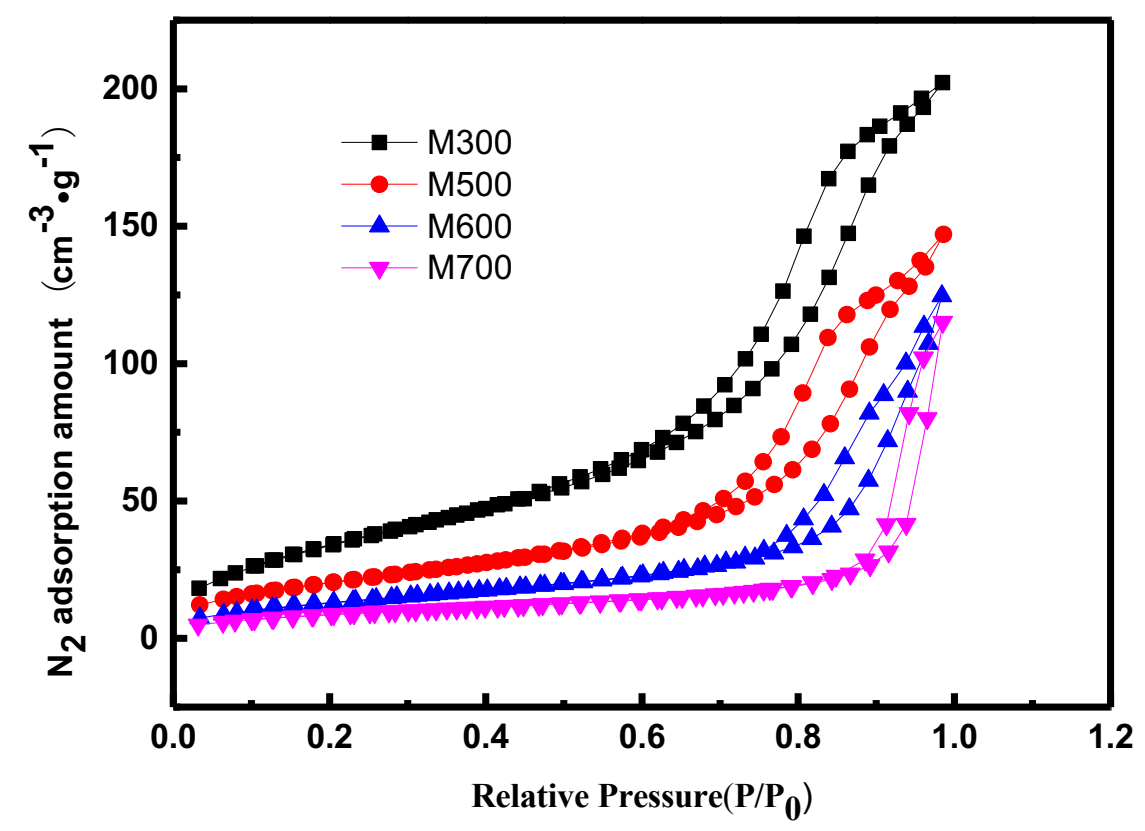

Figure S1. $\mathrm{N}_{2}$ adsorption-desorption isotherms of the mesoporous $\mathrm{TiO}_{2}$ samples after BSA adsorption 


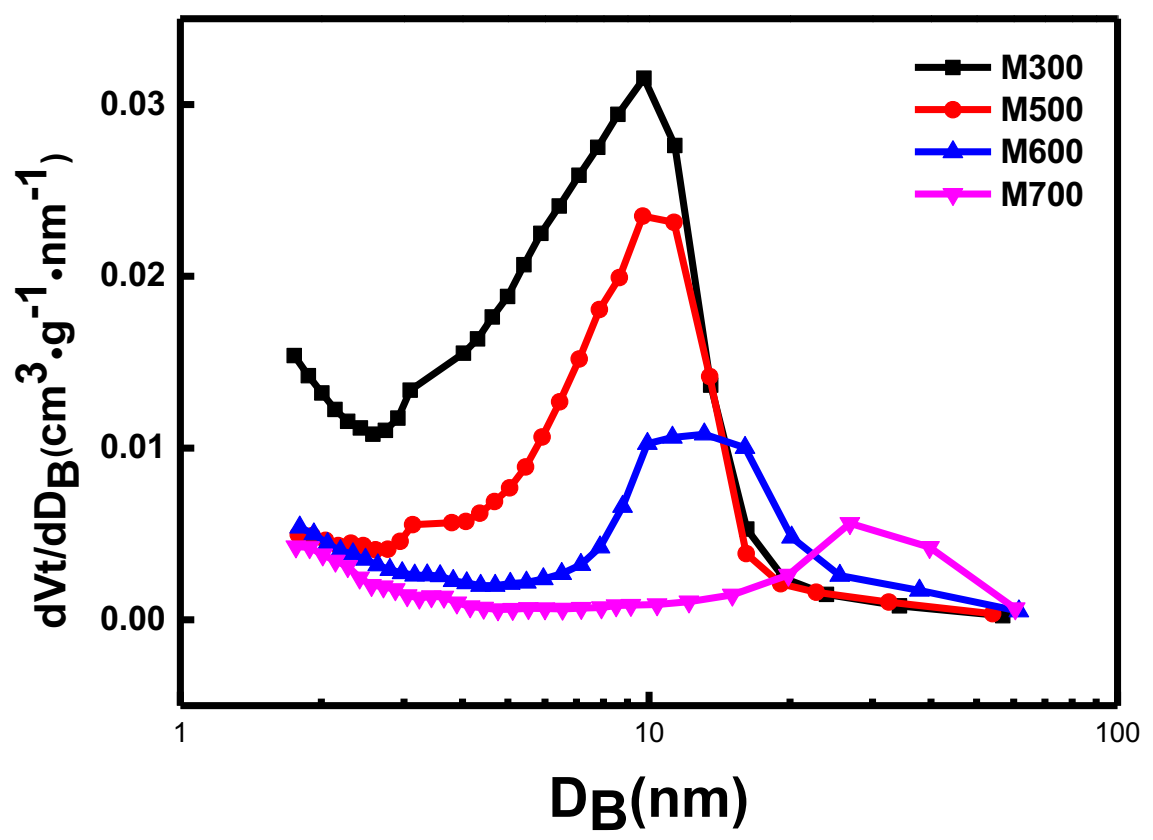

Figure S2. BJH pore size distribution of the mesoporous $\mathrm{TiO}_{2}$ samples after BSA adsorption.

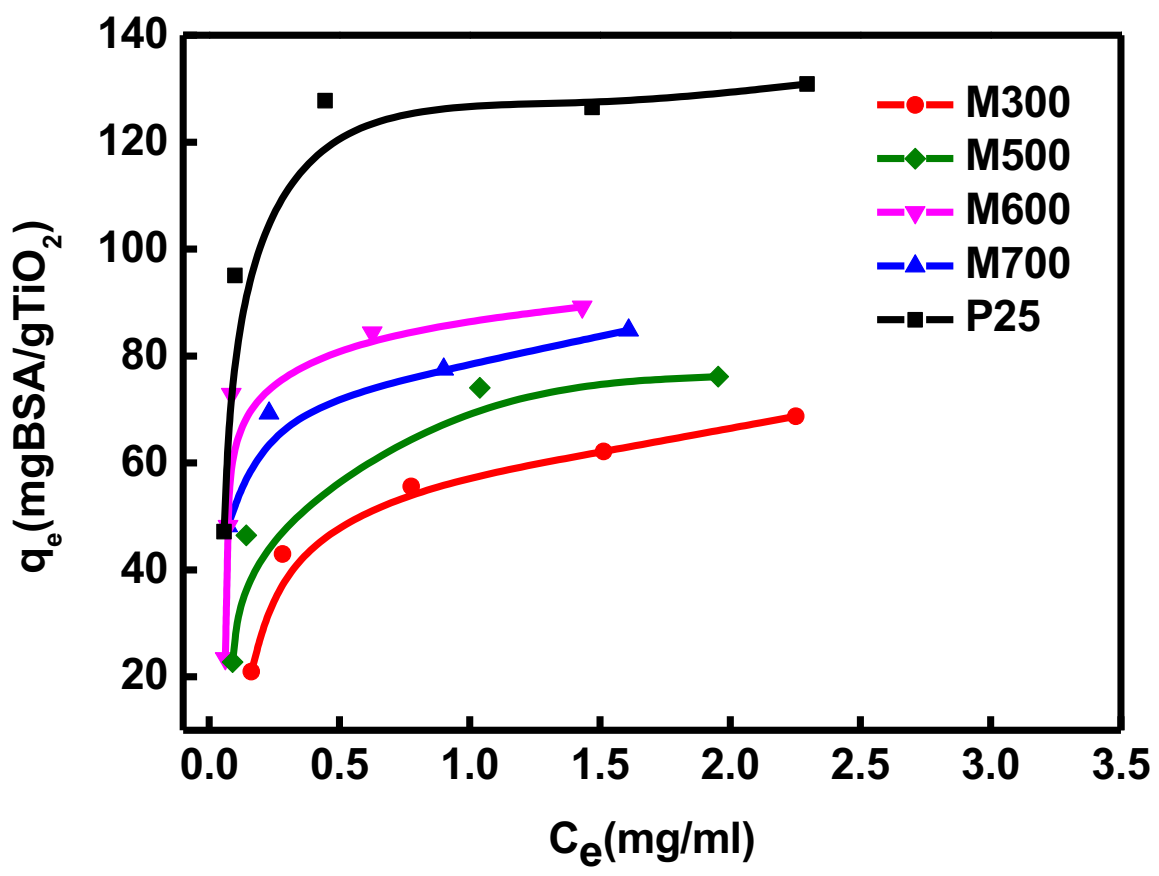

Figure S3. Adsorption isotherm curves of BSA in the studied samples. $\mathrm{q}_{\mathrm{e}}$ : BSA adsorption capacity; $\mathrm{C}_{\mathrm{e}}$ : the equilibrium BSA concentration in the solution. 